\title{
Exogenous GA3 Application Enhances Xylem Development and Induces the Expression of Secondary Wall Biosynthesis Related Genes in Betula platyphylla
}

\author{
Huiyan Guo ${ }^{1,2}$, Yucheng Wang ${ }^{1}$, Huizi Liu ${ }^{1}$, Ping Hu ${ }^{1}$, Yuanyuan Jia ${ }^{1}$, Chunrui Zhang ${ }^{1}$, \\ Yanmin Wang ${ }^{1,3}$, Shan Gu ${ }^{2}$, Chuanping Yang ${ }^{1, *}$ and Chao Wang ${ }^{1, *}$ \\ 1 State Key Laboratory of Tree Genetics and Breeding, Northeast Forestry University, \\ 26 Hexing Road, Harbin 150040, China; E-Mails: wsghy1307@126.com (H.G.); \\ wangyucheng1029@126.com (Y.W.); LHZ249450439@126.com (H.L.); \\ hupingmojiezuo@aliyun.com (P.H.); jiayuayua2015@126.com (Y.J.); zcrlwzy@126.com (C.Z.); \\ wangyanmin1919@aliyun.com (Y.W.) \\ 2 Department of Life Science and Technology, Mudanjiang Normal College, Mudanjiang 157012, \\ China; E-Mail: gushan0731@126.com \\ 3 Forestry Science Research Institute of Heilongjiang Province, Harbin 150081, China \\ * Authors to whom correspondence should be addressed; \\ E-Mails: yangcp@nefu.edu.cn (C.Y.); nefuwangchao@yahoo.com (C.W.); \\ Tel./Fax: +86-451-8219-0607 (C.Y.\& C.W.).
}

Academic Editor: Marcello Iriti

Received: 18 May 2015 / Accepted: 11 September 2015 / Published: 23 September 2015

\begin{abstract}
Gibberellin (GA) is a key signal molecule inducing differentiation of tracheary elements, fibers, and xylogenesis. However the molecular mechanisms underlying the effect of GA on xylem elongation and secondary wall development in tree species remain to be determined. In this study, Betula platyphylla (birch) seeds were treated with 300 ppm GA3 and/or 300 ppm paclobutrazol (PAC), seed germination was recorded, and transverse sections of hypocotyls were stained with toluidine blue; the two-month-old seedlings were treated with $50 \mu \mathrm{M} \mathrm{GA} 3$ and/or $50 \mu \mathrm{M}$ PAC, transverse sections of seedling stems were stained using phloroglucinol- $\mathrm{HCl}$, and secondary wall biosynthesis related genes expression was analyzed by real-time quantitative PCR. Results indicated that germination percentage, energy and time of seeds, hypocotyl height and seedling fresh weight were enhanced by $\mathrm{GA}_{3}$, and reduced by PAC; the xylem development was wider in $\mathrm{GA}_{3}$-treated plants than in the control; the expression of NAC and MYB transcription factors, CESA, PAL, and GA
\end{abstract}


oxidase was up-regulated during $\mathrm{GA}_{3}$ treatment, suggesting their role in GA3-induced xylem development in the birch. Our results suggest that GA3 induces the expression of secondary wall biosynthesis related genes to trigger xylogenesis in the birch plants.

Keywords: Betula platyphylla SUK; GA3; seed germination; xylem development; secondary wall biosynthesis related genes

\section{Introduction}

The expansion of xylem tissue is crucial for plant growth, particularly the growth of woody plants. Therefore, this process is of particular interest to commercial forestry. Unraveling the signaling processes mediating xylem activation and secondary wall development in tree species may shed new light on potential mechanisms to maximize crop yield. During primary growth, the procambium differentiates into vascular tissues including xylem, first forming protoxylem, and vascular cambium develops through secondary growth to form secondary xylem [1]. The present study showed that these processes are controlled by plant hormone regulators including auxin, cytokinin, gibberellins, and ethylene [2].

Gibberellins (GAs) are tetracyclic diterpenoid plant growth hormones that regulate diverse physiological processes including seed germination, stem elongation, leaf expansion, root growth, and the development of reproductive organs [3-5]. GA3 induces the germination of dormant Avena fatua caryopses via regulation of $\mathrm{ABA}$ and ROS-antioxidant status [6]. The seed germination is faster and higher than in control seeds under endogenous $\mathrm{GA}_{3}$ treatment in macaw palm [7]. GA signaling in the cambium has been reported to mediate xylogenesis, and promote fiber elongation [8]. Supplementation with exogenous GA significantly increased the ratio of xylem area to total plant area and increased the proportion of fibers in Arabidopsis [9]. The increase in endogenous $\mathrm{GA}_{3}$ content induces significant increases of xylogenesis in tobacco [10]. Overexpression of the GA receptors PttGID1.1 or PttGID1.3 in aspen stimulates cambial activity to enhance xylem production [8]. The role of signaling processes in the activation of xylem and secondary wall development requires investigation.

It was hypothesized that signals, such as GA, activate the gene-regulated transcriptional network [11]. A previous study found that NTL8 (a membrane-bound NAC transcription factor) mediated the salt regulation of seed germination via GA metabolism in Arabidopsis [12]. A recent study indicated that $S b M Y B 2$ and $S b M Y B 7$ might regulate secondary biosynthesis by GA pathway in transgenic tobacco plants [13]. Transcription of CESA, PAL, and other genes related to cellulose, xylan, and lignin biosynthesis is regulated by the transcription factors NAC and MYB [14-16]. Therefore, we hypothesized that the $N A C, M Y B, C E S A$, and $P A L$ genes of birch plants also controlled the xylem development process regulated by GA.

In this study, in order to investigate the effect of $\mathrm{GA}_{3}$ on the germination of seeds and development of xylem in the birch we monitored the germination, hypocotyl height, and fresh weight of seedlings, measured transverse sections of hypocotyls and seedling stems and analyzed the expression of NAC and MYB transcription factors, CESA, PAL and GA oxidase genes by real-time quantitative PCR (RT-PCR). This work further characterizes the mechanisms of xylem development in birch. 


\section{Results and Discussion}

\subsection{Germination of Birch Seeds}

Open pollinated white birch (B. platyphylla SUK) seeds were moistened with GA3 and/or paclobutrazol (PAC), an inhibitor of $\mathrm{GA}_{3}$ biosynthesis, and water as a control.

Germination was enhanced by $\mathrm{GA}_{3}$ and repressed by PAC. A significantly higher percentage of $\mathrm{GA}_{3}$-treated seeds germinated (93.33\%) than control seeds $(71.33 \%)$, PAC-treated $(54.67 \%)$ or $\mathrm{GA}_{3}+$ PAC-treated seeds $(84.00 \%)$ (Figure 1A). The germination energy of GA3-treated seeds $(72.00 \%)$ was also higher than control seeds $(46.00 \%)$, PAC-treated $(26.67 \%)$ or $\mathrm{GA}_{3}+$ PAC-treated seeds $(61.33 \%)$ (Figure 1B). GA3-treated seeds also germinated faster (6.95 days) than control (8.08 days) or PAC-treated seeds (8.91 days); $\mathrm{GA}_{3}+$ PAC-treated seeds also germinated after roughly seven days (Figure 1C). Seed dormancy and germination are regulated by a number of genes and environmental factors [17]. Various methods have been used to terminate seed dormancy, including hormonal, light and/or temperature treatments [18,19]. Endogenous GAs have previously been used to terminate dormancy and promote seed germination [20]. Our results showed that GA3 promoted germination of birch seeds and ameliorated the impact of PAC on germination.

Application of $\mathrm{GA}_{3}$ significantly induced elongation of birch seedling hypocotyl within the first 15 days of germination (Figure 1D-F). The mean hypocotyl treated with GA3 was $2.13 \mathrm{~cm}$ in length and longer than the control hypocotyls, which measured $0.62 \mathrm{~cm}$ (Figure 1D,E). The fresh weight of seedlings treated with $\mathrm{GA}_{3}$ was $1.65 \mathrm{mg}$, which was also higher than that of control seedlings weighing $0.97 \mathrm{mg}$ (Figure 1F). At $0.5 \mathrm{~cm}$, the hypocotyls of seeds treated with PAC were more stunted than control hypocotyls; the fresh weight of these seedlings was $0.85 \mathrm{mg}$, which was lower than control seedlings (Figure 1D-F), suggesting that the application of PAC had a negative effect on birch seedling hypocotyl growth. However, the growth inhibition induced by PAC was ameliorated by application of $\mathrm{GA}_{3}$, the mean hypocotyl length and the fresh weight of seedlings treated with GA 3 + PAC, respectively, were $1.52 \mathrm{~cm}$ and $1.30 \mathrm{mg}$, further suggesting that $\mathrm{GA}_{3}$ accelerated hypocotyl elongation and seedling growth, consistent with previous studies [21,22].
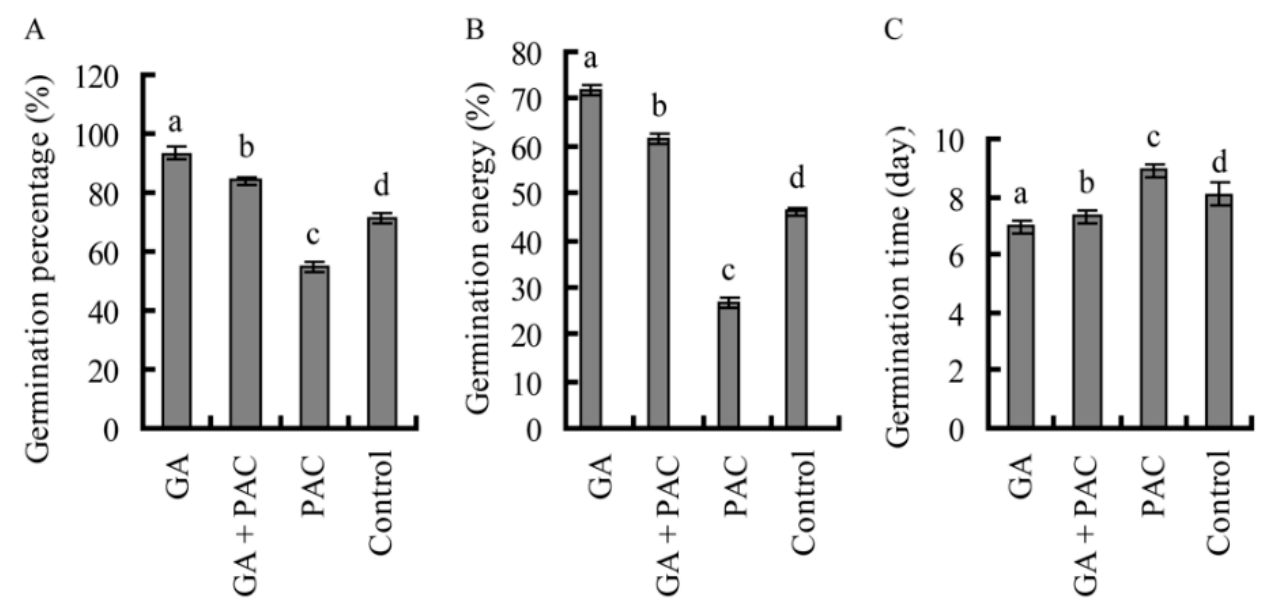

Figure 1. Cont. 

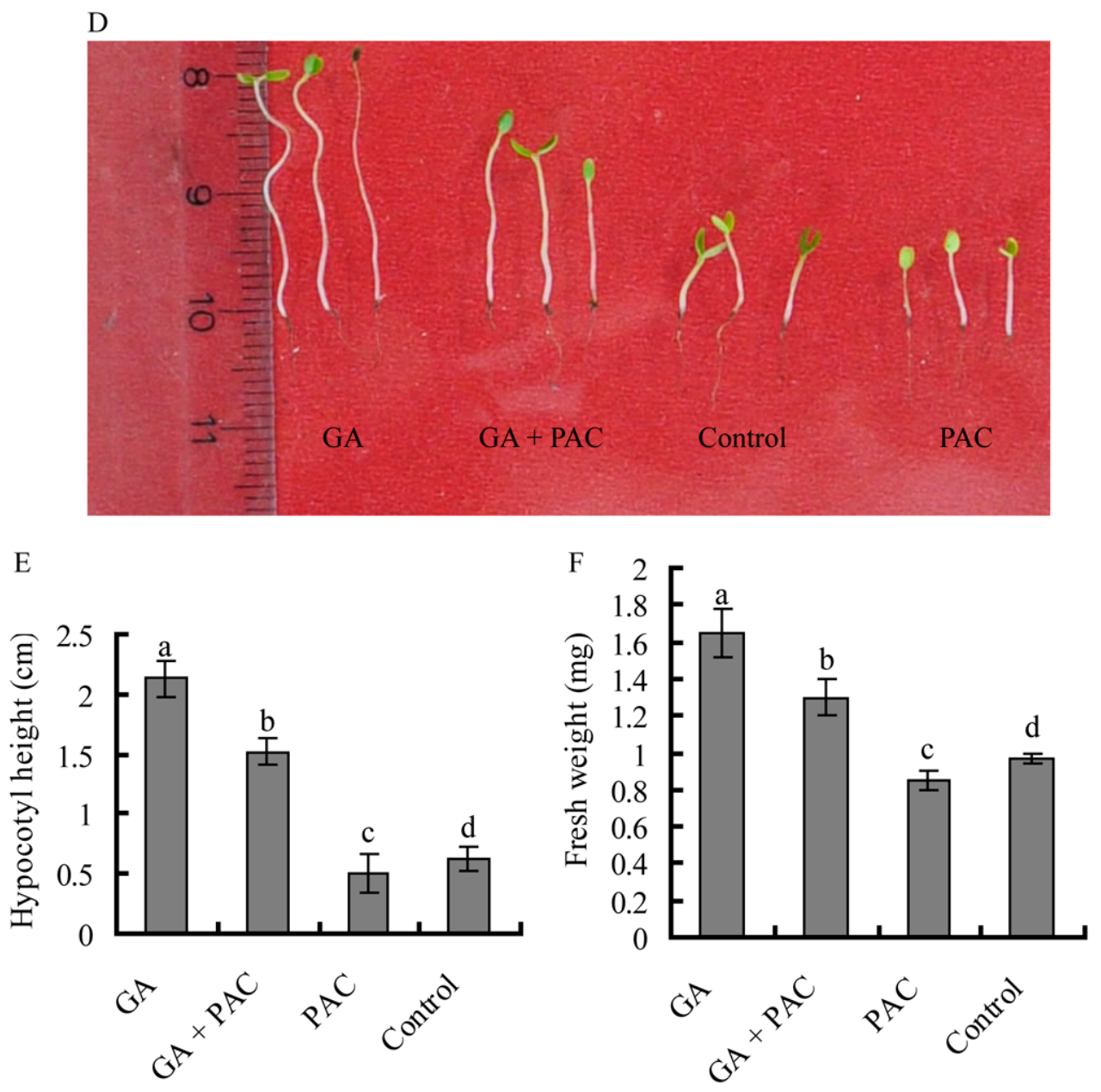

Figure 1. The germination of birch seeds treated with $\mathrm{GA}_{3}$ and/or paclobutrazol (PAC). (A) Germination percentage (\%) of birch seeds treated with $\mathrm{GA}_{3}$ and/or PAC; (B) Germination energy (\%) of birch seeds treated with $\mathrm{GA}_{3}$ and/or PAC; (C) Germination time (day) of birch seeds treated with $\mathrm{GA}_{3}$ and/or PAC; (D) Growth of birch seedlings; (E) Hypocotyl height of birch seedling; and (F) Fresh weight of birch seedlings. Lower case letter indicates $p<0.05$.

\subsection{The Primary Xylem Development of Birch Hypocotyls}

The hypocotyl base was sectioned 15 days after germination and stained with toluidine blue (Figure 2A). Xylem differentiation was observed in hypocotyls treated with $\mathrm{GA}_{3}$ and $\mathrm{GA}_{3}+\mathrm{PAC}_{\text {. }}$ However, differentiation of the primary xylem was weaker in control hypocotyls or hypocotyls treated with PAC than in those treated with $\mathrm{GA}_{3}$ or $\mathrm{GA}_{3}+$ PAC. The diameter of the hypocotyl in $\mathrm{GA}_{3}$ or $\mathrm{GA}_{3}+\mathrm{PAC}$ was wider than that in control; however, it was narrower in PAC than in control (Figure 2B); the number of xylem cell (Figure 2C) in $\mathrm{GA}_{3}$ (about 29) or $\mathrm{GA}_{3}+\mathrm{PAC}$ (about 26) was more than that in control (about 21), PAC (about 16) was the smallest. These results indicate that GA promotes xylem differentiation and primary xylem development. 


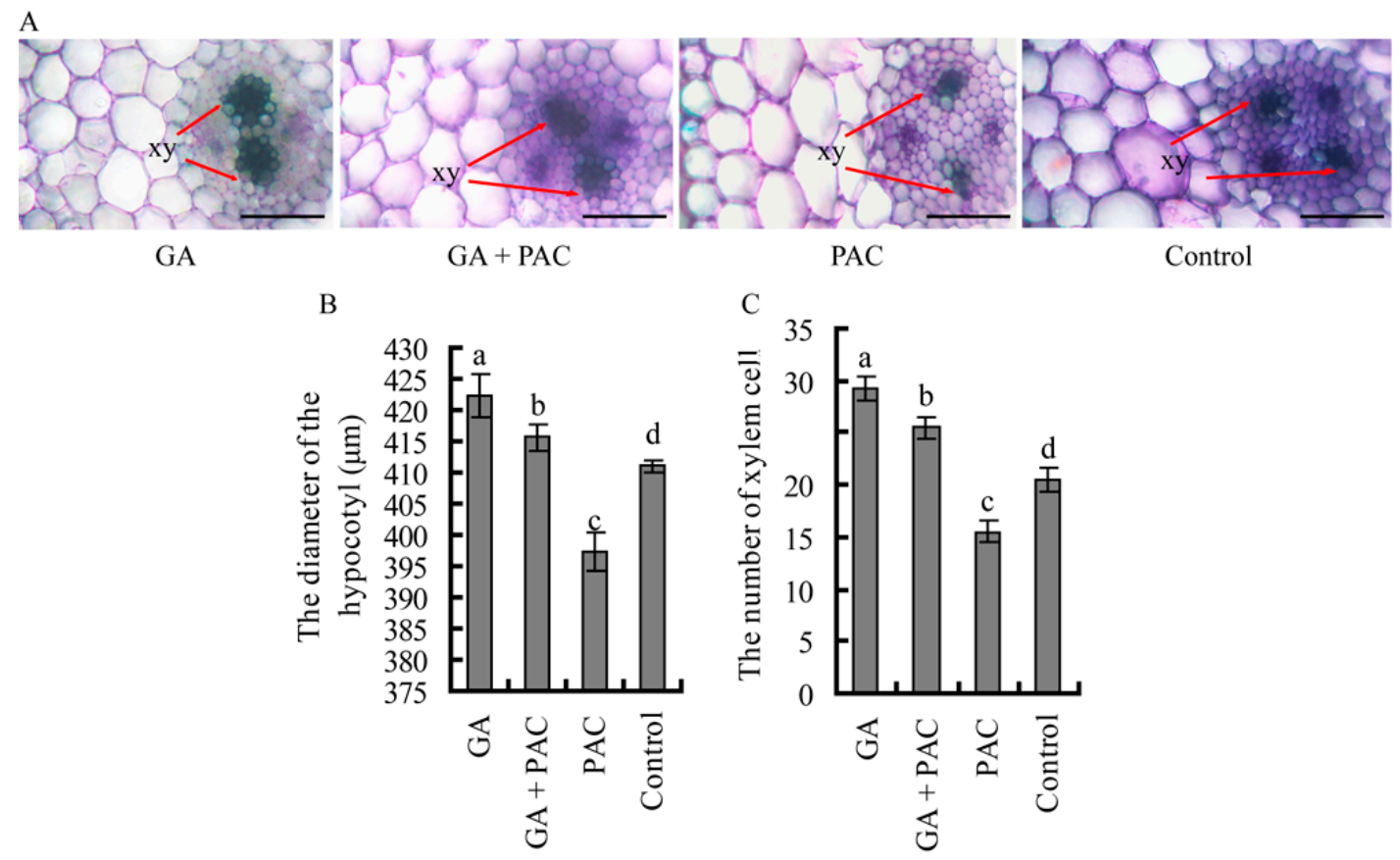

Figure 2. Transverse sections of 15-day-old birch seedlings germinating under $\mathrm{GA}_{3}$ and/or PAC. The hypocotyl base was sectioned 15 days after germination. (A) Toluidine blue staining analysis of the differentiation of xylem. xy, xylem cell. Bars $=50 \mu \mathrm{m}$; (B) The diameter of the hypocotyl $(\mu \mathrm{m})$; and (C) The number of xylem cell. Lower case letter indicates $p<0.05$.

\subsection{Growth and Secondary Xylem Development of Birch Seedlings}

Two-month-old birch seedlings were moistened with $\mathrm{GA}_{3}$ and/or PAC. We found that GA3 and/or PAC had a similar effect on the growth of birch seedlings as in birch hypocotyls. Seedlings treated with $\mathrm{GA}_{3}(36.97 \mathrm{~cm})$ or $\mathrm{GA}_{3}+$ PAC $(33.90 \mathrm{~cm})$ were taller with active apical growth than seedlings treated with water $(21.83 \mathrm{~cm})$ or PAC $(11.03 \mathrm{~cm})$ (Figure 3), in which the apical shoot and apical growth were significantly slower, suggesting that $\mathrm{GA}_{3}$ also promotes stem elongation and apical growth.

After different GA and/or PAC treatments time of two-month-old birch seedling, transverse sections of stems were obtained and stained with phloroglucinol- $\mathrm{HCl}$ to highlight xylem tissue. During development, the area of xylem in the birch stem expanded, and expanded most rapidly in seedlings treated with $\mathrm{GA}_{3}$ or $\mathrm{GA}_{3}+\mathrm{PAC}$; seedlings treated with PAC alone developed less quickly than seedlings treated with water alone (Figure $4 \mathrm{~A}-\mathrm{P}$ ). The ratio of xylem area to total plant area under $\mathrm{GA}_{3}$, $\mathrm{GA}_{3}+\mathrm{PAC}, \mathrm{PAC}$ and control was calculated, respectively (Figure 4Q). The ratio of xylem area to total plant area was slightly higher under $\mathrm{GA}_{3}$ than control and PAC treatments, which were about $18.5 \%$ and $23.3 \%$ in $\mathrm{GA}_{3}, 15 \%$ and $19.2 \%$ in control, and $13.6 \%$ and $17.1 \%$ in PAC, respectively at 3 and 7 day. The ratio of xylem area to total area in plants was significantly higher under $\mathrm{GA}_{3}$ treatment compared with under control condition, but was significantly lower under PAC treatment at 14 and 21 day. The ratio was $47.1 \%$ in $\mathrm{GA}_{3}, 37.9 \%$ in control and $20.9 \%$ in $\mathrm{PAC}$ at 14 day, and $77.5 \%$ in $\mathrm{GA}_{3}, 61.7 \%$ in control and $40 \%$ in PAC at 21 day. 


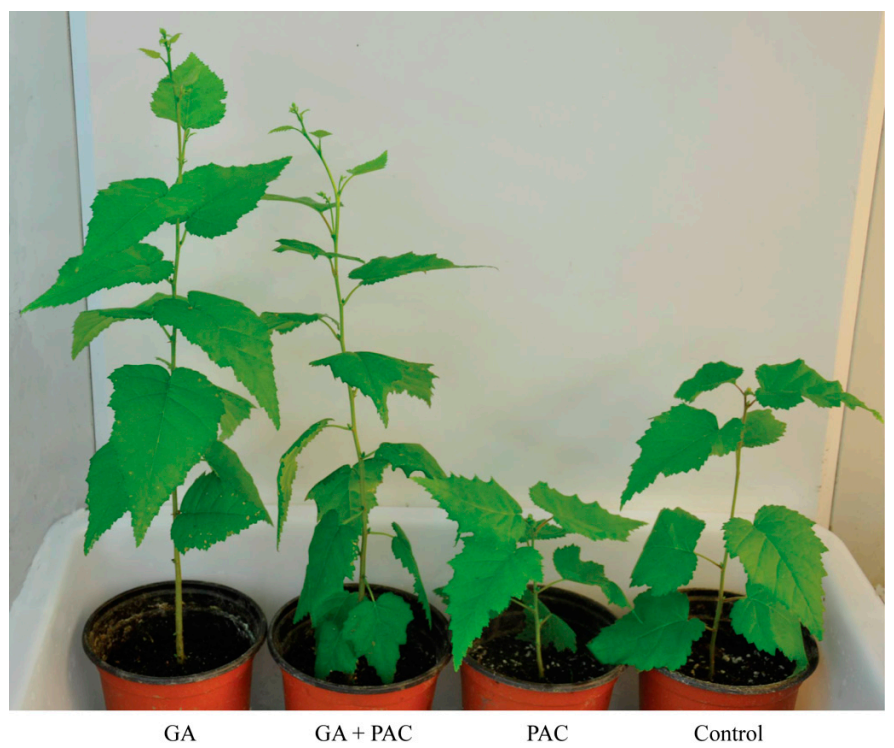

Figure 3. Phenotypic changes of 2-month-old birch seedlings under $\mathrm{GA}_{3}$ and/or PAC.

The GA3 pathway promotes xylogenesis in many species [23,24]. In Fraxinus mandshurica Rupr. var. japonica Maxim. seedlings, the width of xylem in stems was enhanced by GAs (GA3 and GA4) [23]. In Zinnia elegans, a slight increase in the frequency of tracheary element (TE) differentiation and a substantial increase in lignin content were observed under $\mathrm{GA}_{3}$ treatment [23]. PAC treatment probably inhibited xylem development by inhibiting GA synthesis, as the growth deficiency was rescued by exogenous application of $\mathrm{GA}_{3}$ [4]. Our results (Figure 4) further demonstrate that $\mathrm{GA}_{3}$ is an important factor in regulating xylem development of birch plants, consistent with previous studies.

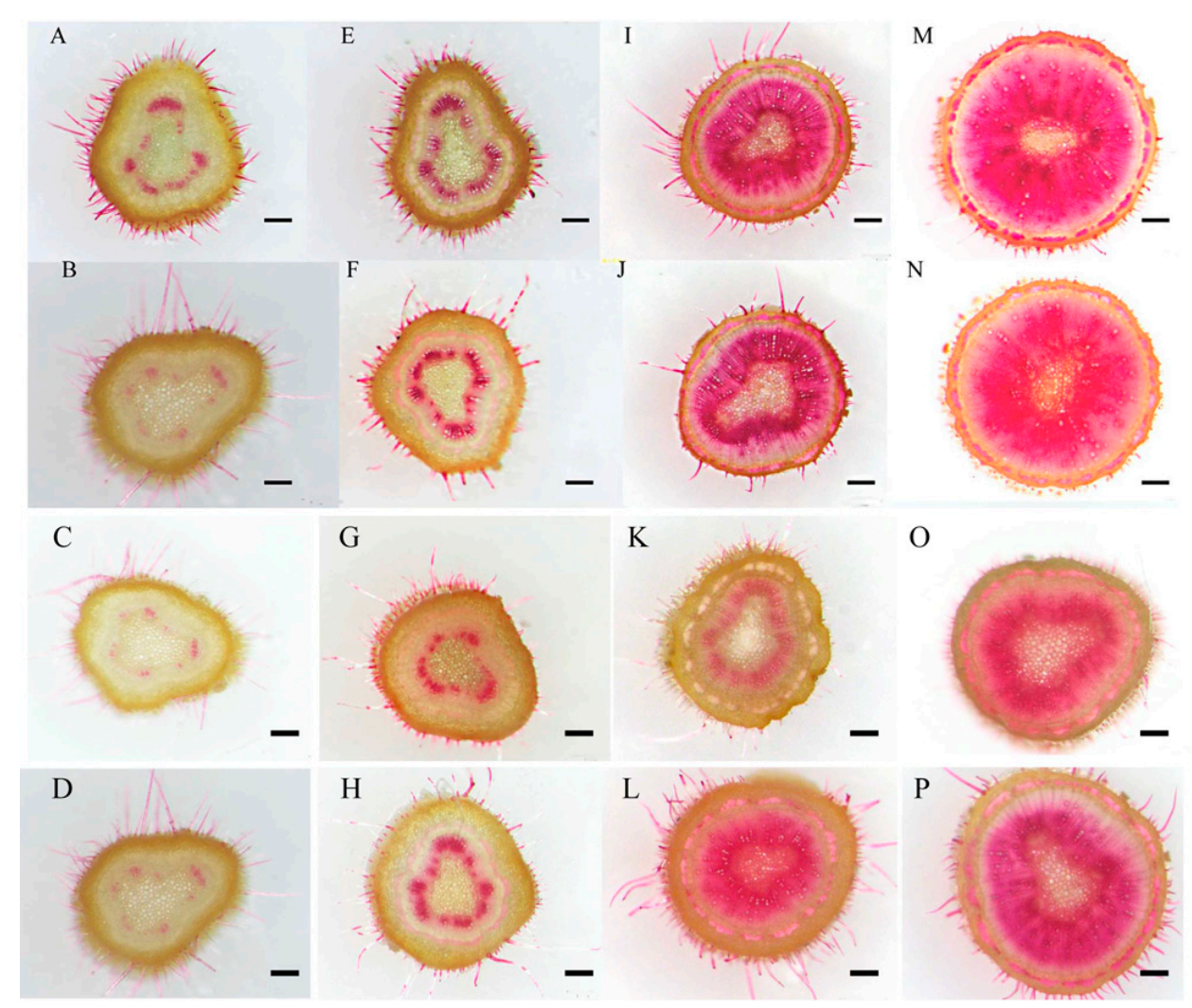

Figure 4. Cont. 


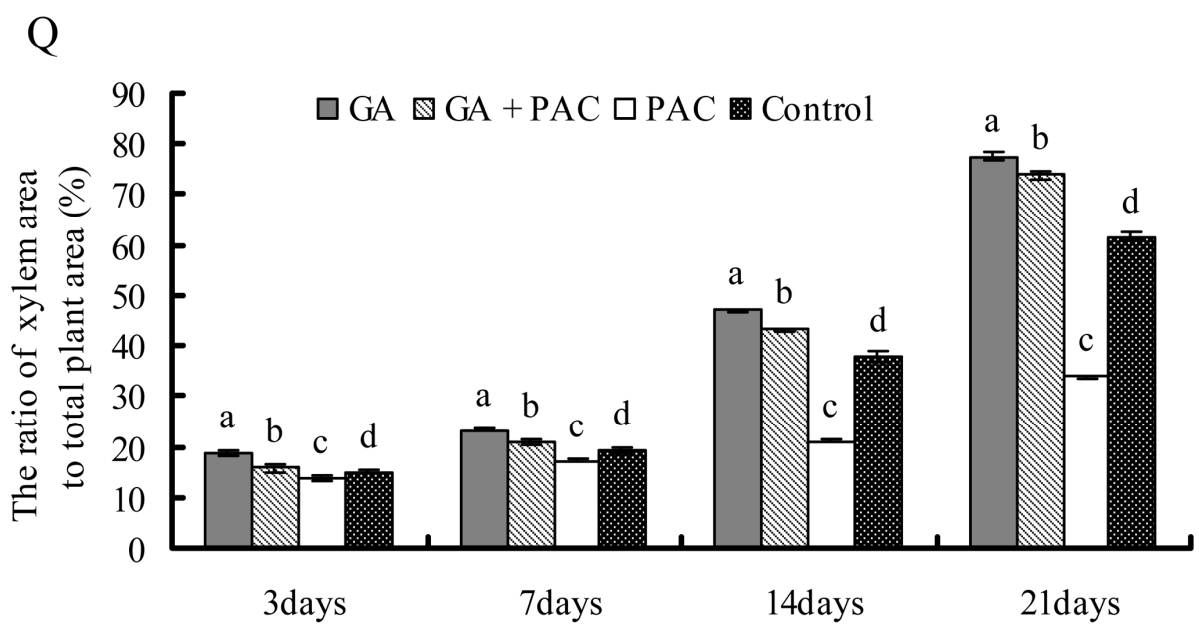

Figure 4. Transverse sections of 2-month-old birch seedlings and the ratio of xylem area to total area under GA3 and/or PAC treatments. (A-D) GA and/or PAC treatment for 3 days; (E-H) GA and/or PAC treatment for 7 days; (I-L) GA and/or PAC treatment for 14 days; and (M-P) GA and/or PAC treatment for 21 days. (A,E,I,M) GA treatment; $(\mathbf{B}, \mathbf{F}, \mathbf{J}, \mathbf{N})$ GA + PAC treatment; $(\mathbf{C}, \mathbf{G}, \mathbf{K}, \mathbf{O})$ PAC treatment; and $(\mathbf{D}, \mathbf{H}, \mathbf{L}, \mathbf{P})$ water treatment (control). Phloroglucinol- $\mathrm{HCl}$ was used to stain lignin to highlight xylem vessels and fibers. Bars $=1 \mathrm{~mm} ;(\mathbf{Q})$ The ratio of xylem area to total area in birch plants under $\mathrm{GA}_{3}$, $\mathrm{GA}_{3}+\mathrm{PAC}$, PAC and control for 3, 7, 14 or 21 days. Error bars were obtained from multiple replicates of the real-time PCR. Lower case letter indicates $p<0.05$.

\subsection{Gene Selection and Expression Pattern under GA3 Treatment}

In a previous study [25], differential gene expression (DGE) indicated that $4 N A C, 5 M Y B, 2 C E S A$, and 2 PAL genes were altered with higher RPKM values among tension wood (TW), opposite wood (OW) and normal wood (NW), suggesting that these genes may play a role in regulating xylem development in the birch. Therefore, these potential secondary cell wall (SCW) genes were selected for expression analysis in this study.

The expression of 4 NAC, 5 MYB, 2 CESA, 2 PAL, and 2 GA20ox genes related to xylem development obtained using the DGE and bioinformatics analysis in seedlings treated with GA3 and/or PAC was analyzed by real-time quantitative PCR. The expression of most genes was up-regulated on days 7, 14, and 21 in plants treated with $\mathrm{GA}_{3}$ or $\mathrm{GA}_{3}+\mathrm{PAC}$; the expression of almost all genes was down-regulated in plants treated with PAC, on days 7, 14, and 21 compared with plants treated with water alone (Figure 5).

Differentiation of vascular cambium into xylem mother cells is regulated by plant hormones. The coordinated activation of secondary wall biosynthesis genes during wood formation is mediated by a transcriptional network encompassing secondary wall NAC and MYB master switches and their downstream transcription factors [26]. Our results suggest that $\mathrm{GA}_{3}$ induced the expression of 4 NAC and 5 MYB transcription factors, and the 2 CESA, $2 P A L$, and 2 GAoxidase genes, suggesting that birch SCW transcription factors respond to GA3. Genes, such as NAC2, CESA4, PAL3, GA20ox1, and GA20ox3, were down-regulated at 3 day in plants treated with $\mathrm{GA}_{3}$ or $\mathrm{GA}_{3}+\mathrm{PAC}$ compared with control. However, these genes were up-regulated during the treatment period resulting in altered xylem phenotype, indicating that the treatments were long enough to modify the phenotypes and affect 
the genes expression via direct or indirect pathway. The temporary decrease and subsequent increase of gene expression suggests complex molecular mechanisms of gene regulation.

NAC transcription factors are the key regulators of secondary wall synthesis. Specific loss of secondary walls was observed at the valve margins of Arabidopsis in NST1 mutants, indicating that NST1 mediated secondary wall biosynthesis [27]. GA3 treatment of birch seedlings up-regulated the expression of BplNAC1 and BplNAC2 (Figure 5). Previous reports suggested that $N A C$ genes were up-regulated by some hormones [28]. OsNAC2 affected plant height by regulating the GA pathway in rice [29]. In this study, xylem development was promoted by $\mathrm{GA}_{3}$, and expression of BplNAC1 and BplNAC2 genes, which are closely related to SND2 gene (AtNAC073) and NST1 gene (AtNAC043) of Arabidopsis, were up-regulated by GA3. AtNAC073 belongs to the SND2 family, related to genes involved in secondary cell wall development and promotion of fiber cell area [30]. Taken together, our results indicate that BpINAC1 and BplNAC2 are involved in xylem development, respond to the GA signal pathway, and were induced by GA3.
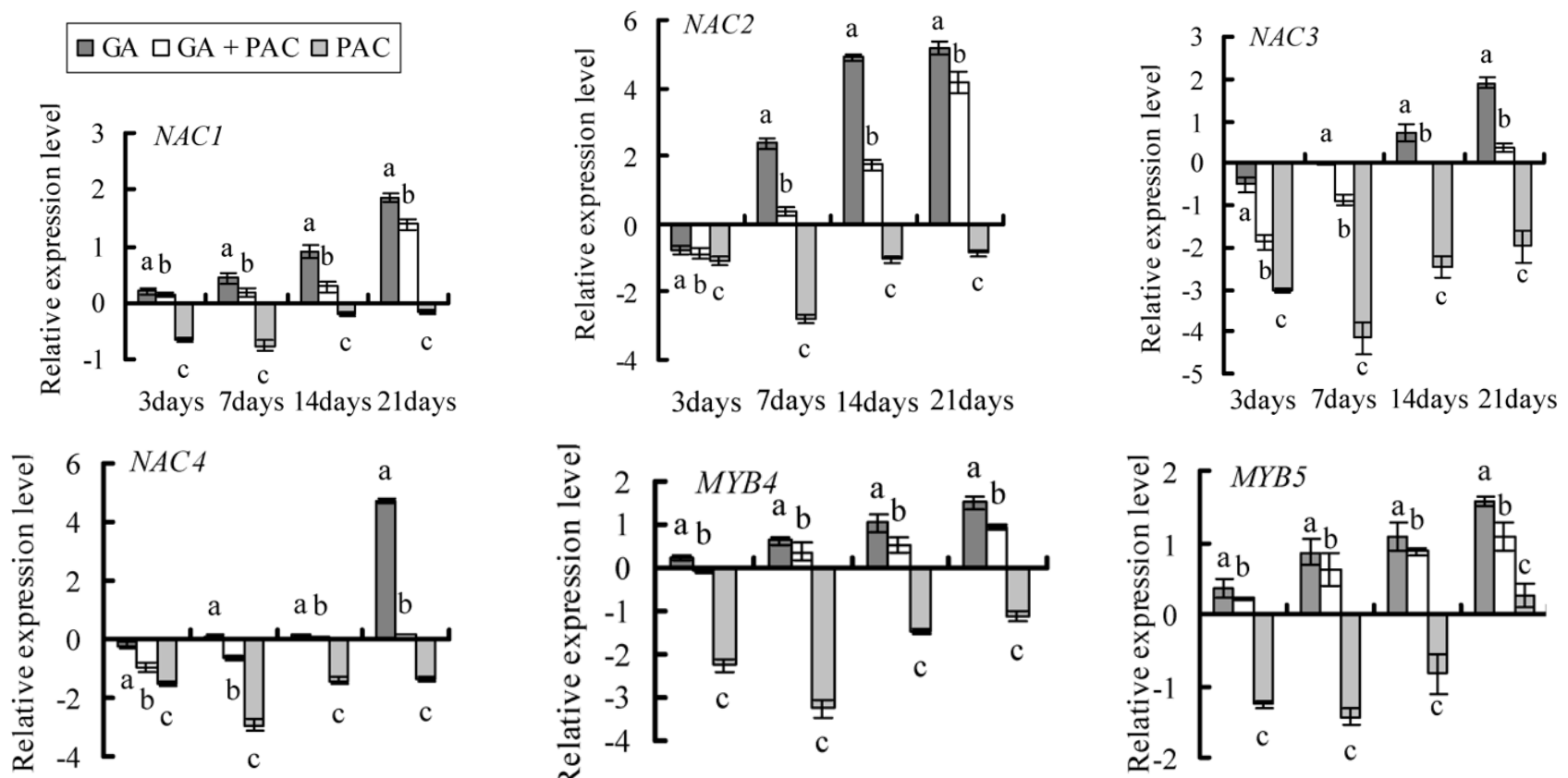

3days 7days 14days 21days
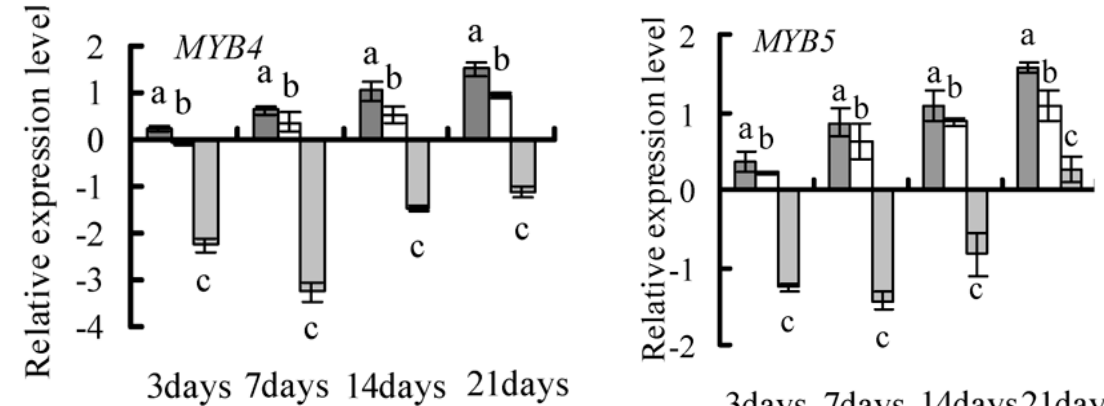

3days 7days 14days 21 days
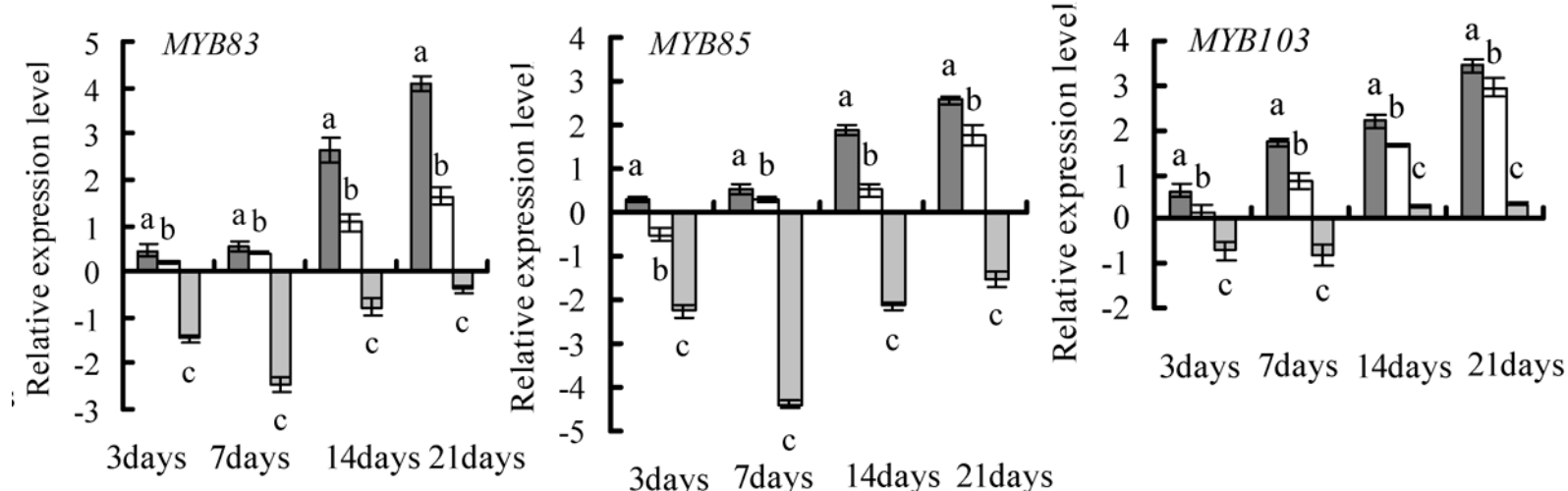

Figure 5. Cont. 


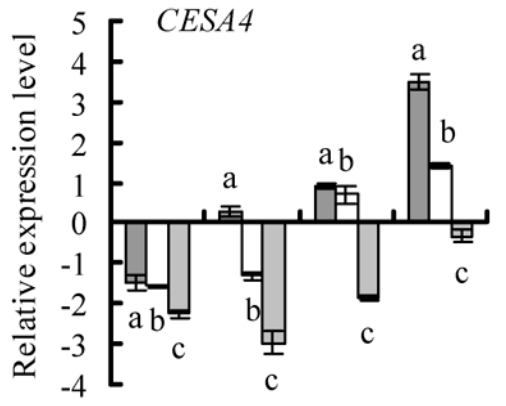

3days 7days 14days 21days

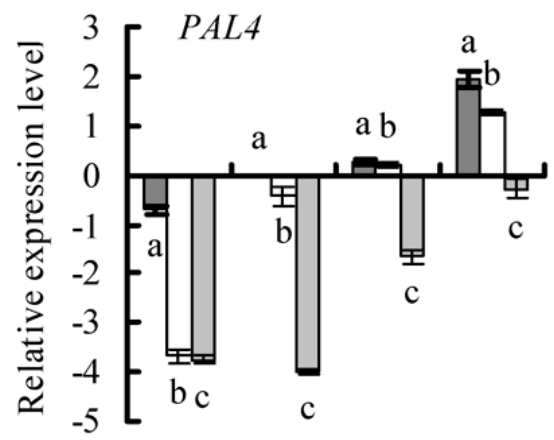

3days 7days 14days 21days

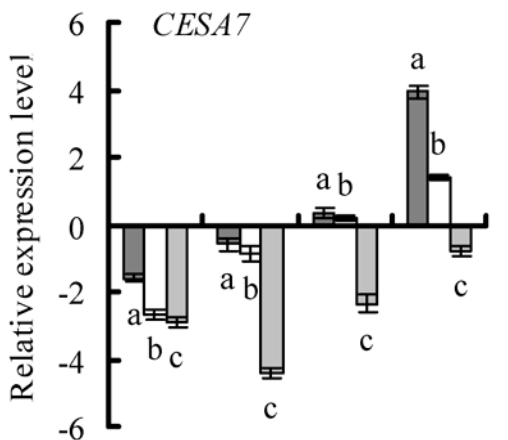

3days 7days 14days 21days

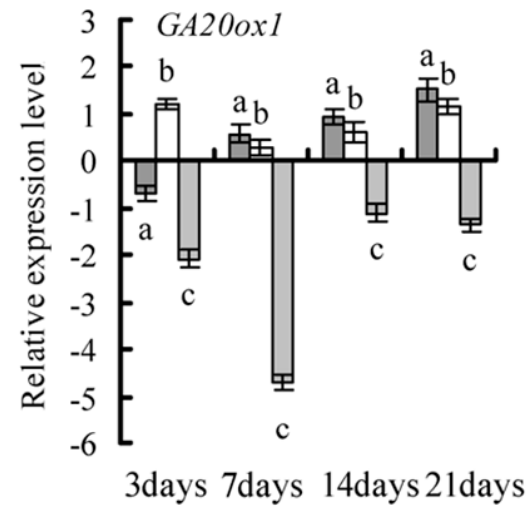

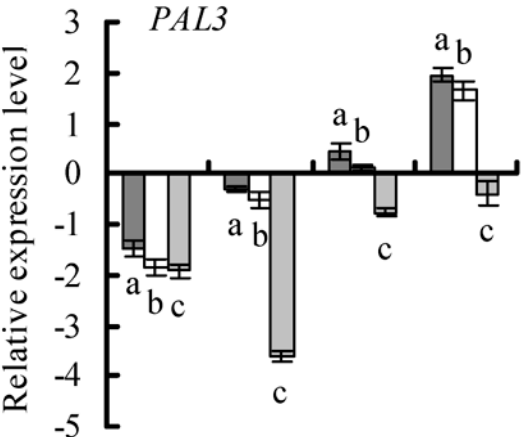

3days 7days 14days 21days

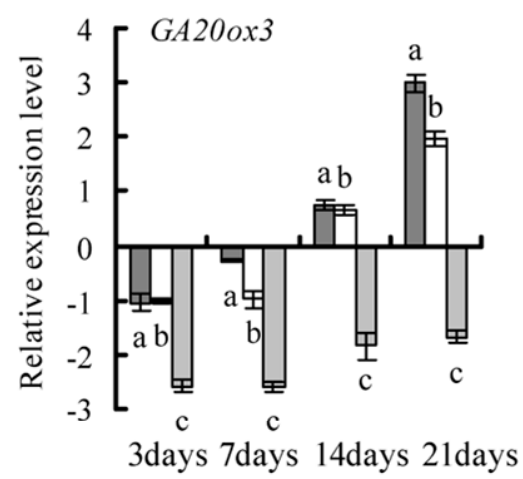

Figure 5. Expression analysis of the genes in birch plants under $\mathrm{GA}_{3}$ and/or PAC. These genes include $N A C, M Y B, P A L, C E S A$, and GA20ox. Error bars were obtained from multiple replicates of the real-time PCR. Lower case letter indicates $p<0.05$.

In general, the MYB transcription factors downstream of SND1, are regulated by SND1. We found that BplMYB83 is similar to AtMYB46 and AtMYB83, and BplMYB103 is similar to AtMYB103 of Arabidopsis in protein sequence. Both $M Y B$ genes of birch were induced by $\mathrm{GA}_{3}$, but suppressed by PAC during birch seedling growth (Figure 5). AtMYB46 and AtMYB83 are directly activated by SND1 and act as molecular switches in the SND1-mediated transcriptional network during secondary wall deposition [31,32]. AtMYB103 was previously reported to regulate secondary cell wall formation [33]. The expression of GAMYB was induced by GA, and is a component of the GA-responsive pathway, leading to GA-inducible gene expression during seed germination in barley [34]. We found that GA3 promoted xylem development and that BplMYB83, BplMYB5, and BplMYB52 were up-regulated by $\mathrm{GA}_{3}$, suggesting that these genes promoted secondary xylem development in birch.

NAC and MYB transcription factors regulate the biosynthesis of secondary cell wall components including cellulose and lignin. MYB is reported to bind promoters of cellulose and lignin synthesis genes, such as CESAs and PAL, and induce their expression [35,36]. In this study, BplCESA4 and BplCESA7 were up-regulated in GA3-treated seedlings. CESAs are critical to xylem development and are necessary for synthesis of secondary cell wall in plants [37,38]. BplCESA4 and BplCESA7 share homology with AtCESA4, and AtCESA7, which were implicated in secondary cell wall biosynthesis in Arabidopsis $[39,40]$. The DGE and sequence analysis suggested that BplCESA4 and BplCESA7 may be associated with xylem development and deposition of secondary cell wall in birch. Germination and embryonic axis growth in Medicago truncatula are inhibited by ABA, and the expression of the CESAs 
and other cell-wall loosening and expansion genes were also down-regulated by ABA treatment [41]. In Eucalyptus tereticornis, all three EtCESA transcripts were also up-regulated by GA [42], suggesting that CESA expression is influenced by hormones. In this study, GA promotion of hypocotyl and xylem expansion may be attributed to the induction of genes involved in cell wall biosynthesis, including CESAs. The expression of BplPAL3 and BplPAL4 was also up-regulated by GA3. BplPAL3 has sequence similarity with AtPAL1 and AtPAL2. Based on analysis of the EST data, the AtPALs are likely candidates for lignification, or overlapping PAL metabolic networks, which are operative in vascular tissues [43]. These results suggest that the two birch $P A L$ genes were involved in GA-induced birch xylem development.

BplGA20ox1 and BplGA20ox3 are homologous to AtGA20ox of Arabidopsis. GA 20-oxidase is an enzyme that catalyzes the last three steps in the synthesis of active GAs and is a potential control point in the regulation of GA biosynthesis [44]. GA20ox is also induced by many hormones [45]. AtGA20ox1 and AtGA20ox 2 promote hypocotyl and internode elongation [46]. PtGA20ox was recently reported to be highly expressed in the mature xylem of vascular tissues, and directly mediates secondary xylem formation [47]. In this study, the expression of birch BplGA20oxl was inhibited by PAC, resulting in shorter stem and slower growth. $\mathrm{GA}_{3}$ induced longer stems and faster growth. A previous study found that GA20ox2 transcription was noticeably reduced following GA application within 15 min of hormone treatment in gal-3 mutant Arabidopsis [48]; The expression of AtGA20ox2 and AtGA20ox3 was up-regulated initially, and then down-regulated by exogenous GA4 in gal-3 seeds [49]. In our study, the expression of GA20ox1 and GA20ox3 was transiently down-regulated, and then up-regulated, which may be due to the fact that the treatments were long enough and further implied that the GA20ox mediates the endogeous GA content regulation at an early stage and promotes the growth and development of birch through the direct or indirect pathway.

\section{Experimental Section}

\subsection{Seed Germination and Treatment}

Open pollinated Northeast White Birch (B. platyphylla) seeds were collected and stored in a sealed plastic box at $4{ }^{\circ} \mathrm{C}$. The seeds were rinsed thoroughly with tap water prior to germination in plastic pots and subsequently incubated at $30^{\circ} \mathrm{C}$. Germinated seeds were moistened every second day with $300 \mathrm{ppm}$ GA containing $0.1 \%(v / v)$ Tween-80 and $0.1 \%(v / v)$ ethanol and/or 300 ppm PAC (an inhibitor of GA biosynthesis), or water containing $0.1 \%(v / v)$ Tween-80 and $0.1 \%(v / v)$ ethanol as control. After seven days, only water was used to moisten all seeds. Fifty seeds were incubated under each experimental condition, and all experiments were carried out in triplicate. The number of germinated seeds was recorded every day for 15 days and seeds were considered germinated when the emerging radicle was approximately $2 \mathrm{~mm}$ long. On day 15 the percentage of germinated seeds (germination percentage), germination energy, and time to germination (germination time) were calculated when no further germination occurred for three days in all treatments. Hypocotyl height was recorded and fresh seedlings were also weighed on day 15 after germination. The hypocotyls of germinated seeds were collected and fixed in FAA solution (50\% ethanol, 5\% glacial acetic acid, and 5\% formaldehyde) for anatomical analysis. 


$$
\begin{gathered}
\text { Germination Percentage }=\frac{\text { the Number of Germination Seeds }}{\text { the Number of Total Seeds }} \times 100 \% \\
\text { Germination Energy }=\frac{\text { the Number of Germinarion Seeds to Peak }}{\text { the Number of Total Seeds }} \times 100 \%
\end{gathered}
$$

$$
\text { Germination Time }=\frac{\sum\left(G_{i} \times D_{i}\right)}{\sum G_{i}}
$$

$G_{i}$ represents the number of germinated seeds on day $i$, and $D_{i}$ denotes the number of days taken for germination.

\subsection{Seedling Growth and Treatment}

Birch seeds were sown in plastic pots, and incubated under a 16/8 h day/night $\left(24 / 22{ }^{\circ} \mathrm{C}\right)$ at a $60 \%-70 \%$ relative humidity and $400 \mu \mathrm{mol} \cdot \mathrm{m}^{-2} \cdot \mathrm{s}^{-1}$ light intensity in a greenhouse. Two months later, seedlings grew in pots that had achieved the same height and were sprayed every second day with $50 \mu \mathrm{M}$ $\mathrm{GA}_{3}$ and/or $50 \mu \mathrm{M}$ PAC solution or water for 21 days. The stems of birch seedlings were harvested separately on days $3,7,14$, or 21 after $\mathrm{GA}_{3}$ and/or PAC treatment. Six seedlings were used under each treatment at each harvest time, and three biological replicates were used for each experiment (in total 18 seedlings per condition). The basal stems of three seedlings in each condition were fixed in FAA solution (70\% ethanol, 5\% glacial acetic acid, and 5\% formaldehyde) and used for anatomical analysis. The stems of the other three seedlings were immediately frozen in liquid nitrogen and stored at $-80{ }^{\circ} \mathrm{C}$ for RNA extraction, which was then used for RT-PCR analysis.

\subsection{Histological Analysis}

To visualize xylem development in hypocotyls (at 15 days post-germination) and birch seeding stems (at least two months post-germination), the bases of hypocotyls were cut into about $50-\mu \mathrm{m}$ sections by hand. The fifth internodes from base to tip of the two-month-old birch stems after 21 days treatment under GA3, GA + PAC, PAC, and control were cut into about 50- $\mu \mathrm{m}$ sections manually. Hypocotyl and stem cross-sections were stained with $0.025 \%$ toluidine blue or $5 \%$ phloroglucinol- $\mathrm{HCl}$.

The diameter of the hypocotyl $(\mu \mathrm{m})$ and the number of the xylem cell were measured from sections stained using toluidine blue; the ratio of xylem area to total area was measured from sections stained with phloroglucinol-HCl for each genotype using ImageJ software (Available online: http://rsbweb.nih.gov/ij/), in triplicate.

\subsection{Gene Selection and Relative Expression of the SCW Genes by qRT-PCR}

Birch SCW genes were selected using DGE analysis of birch transcriptome data [25]. The abundance of each gene was determined by calculating the reads per kb per million reads (RPKM) as described by the previous method [50]. GA oxidase genes were selected from the birch genome (Available online: http://birch.genomics.cn/).

Total RNA from the first to the fourth internode of each birch stem was extracted using the cetyltrimethylammonium bromide $(\mathrm{CTAB})$ method. For real-time quantitative PCR analysis, total 
RNA was treated with DNase I and used for first-strand cDNA synthesis using PrimeScript ${ }^{\mathrm{TM}}$ RT reagent Kit (TaKaRa, Osaka, Japan). Real-time quantitative RT-PCR was performed with SYBR Premix Ex TaqTM, and primers were designed by primer 5 software (Premier, Palo Alto, CA, USA) (Table S1). $N A C, M Y B, C E S A$ and $P A L$ genes from the birch tension wood (TW), opposite wood (OW) and normal wood (NW) transcriptome [25], and GA20ox genes from the birch genome (Available online: http://birch.genomics.cn/) were subjected to RT-PCR analysis under the following conditions: $94{ }^{\circ} \mathrm{C}, 30 \mathrm{~s}$; 45 cycles at $94{ }^{\circ} \mathrm{C}$ for $12 \mathrm{~s}, 58^{\circ} \mathrm{C}$ for $30 \mathrm{~s}, 72^{\circ} \mathrm{C}$ for $45 \mathrm{~s} ; 79^{\circ} \mathrm{C}, 1 \mathrm{~s}$ for plate reading. A melting curve was generated for each sample to assess the purity of the amplified products. The relative mRNA levels were determined by normalizing the PCR threshold cycle number of each gene to that of $\alpha$-tubulin (GenBank number: FG067376) and ubiquitin (GenBank number: FG065618) reference genes. Expression levels were calculated from the threshold cycle using the delta-delta CT method [51]. Three biological replicates were used in the experiments.

\subsection{Statistical Analysis}

Analysis of variance (ANOVA) of germination percentage, germination energy, germination time, hypocotyl height, fresh weight, stem height, the diameter of hypocotyl, the ratio of xylem area to total area of birch seedlings, and the relative expression of genes using qRT-PCR was performed using SPSS software (SPSS, Chicago, IL, USA). DUNCAN method was used to perform differential analyses by comparing these experiments. The level of significance was set at $p<0.05$.

\section{Conclusions}

The effect of $\mathrm{GA}_{3}$ on germination of seeds, xylem development, and secondary wall biosynthesis related genes expression in B. platyphylla was studied. $\mathrm{GA}_{3}$ treatment increased the germination percentage, germination energy and germination time of seeds, hypocotyl height, and fresh weight of seedlings. Transverse sections of hypocotyls of germinated seed and stems of seedlings showed that $\mathrm{GA}_{3}$ increased xylem development while PAC suppressed it. Application of GA3 entirely ameliorated the phenotype of PAC treatment. GA 3 treatment induced the expression of MYB and NAC transcription factors, $C E S A, P A L$, and $G A$ oxidase genes. These results suggest that birch seed germination and xylem development is promoted by $\mathrm{GA}_{3}$. Birch SCW genes are induced by $\mathrm{GA}_{3}$ and facilitate xylem development in response to $\mathrm{GA}_{3}$.

\section{Supplementary Materials}

Supplementary materials can be found at http:/www.mdpi.com/1422-0067/16/09/22960/s1.

\section{Acknowledgments}

This work was supported by the National High Technology Research and Development Program ("863" Program) of China (2011AA100202) and the Innovation Project of State Key Laboratory of Tree Genetics and Breeding (Northeast Forestry University, 2013B02). 


\section{Author Contributions}

Conceived and designed the experiments: Huiyan Guo, Chuanping Yang and Chao Wang; Performed the experiments: Huiyan Guo, Huizi Liu, Chunrui Zhang, Yuanyuan Jia and Ping Hu; Analyzed the data and contributed reagents/materials/analysis tools: Huiyan Guo, Yanmin Wang, Chao Wang and Shan Gu; Wrote the paper: Huiyan Guo, Yucheng Wang and Chao Wang; Edited the draft manuscript: Huiyan Guo, Yucheng Wang, Chuanping Yang and Chao Wang.

\section{Conflicts of Interest}

The authors declare no conflict of interest.

\section{Abbreviations}

GA: gibberellic acid; PAC: paclobutrazol; CESA: cellulose synthase; PAL: phenylalanine ammonialyase; SCW genes: secondary cell wall biosynthetic genes; SND: secondary wall-associated NAC domain; SWNs: secondary wall NAC domain transcription factors; NST: NAC secondary wall thickening-promoting factor.

\section{References}

1. Nieminen, K.M.; Kauppinen, L.; Helariutta, Y. A weed for wood? Arabidopsis as a genetic model for xylem development. Plant Physiol. 2004, 135, 653-659.

2. Milhinhos, A.; Miguel, C.M. Hormone interactions in xylem development: A matter of signals. Plant Cell Rep. 2013, 32, 867-883.

3. Ayano, M.; Kani, T.; Kojima, M.; Sakakibara, H.; Kitaoka, T.; Kuroha, T.; Angeles-Shim, R.B.; Kitano, H.; Nagai, K.; Ashikari, M. Gibberellin biosynthesis and signal transduction is essential for internode elongation in deepwater rice. Plant Cell Environ. 2014, 37, 2313-2324.

4. Ribeiro, D.M.; Araujo, W.L.; Fernie, A.R.; Schippers, J.H.; Mueller-Roeber, B. Translatome and metabolome effects triggered by gibberellins during rosette growth in Arabidopsis. J. Exp. Bot. 2012, 63, 2769-2786.

5. Schwechheimer, C; Willige, B.C. Shedding light on gibberellic acid signalling. Curr. Opin. Plant Biol. 2009, 12, 57-62.

6. Cembrowska-Lech, D.; Koprowski, M.; Kepczynski, J. Germination induction of dormant Avena fatua caryopses by $\mathrm{KAR}_{1}$ and $\mathrm{GA}_{3}$ involving the control of reactive oxygen species $\left(\mathrm{H}_{2} \mathrm{O}_{2}\right.$ and $\mathrm{O}_{2} \cdot{ }^{-}$) and enzymatic antioxidants (superoxide dismutase and catalase) both in the embryo and the aleurone layers. J. Plant Physiol. 2015, 176, 169-179.

7. Bicalho, E.M.; Pinto-Marijuan, M.; Morales, M.; Muller, M.; Munne-Bosch, S.; Garcia, Q.S. Control of macaw palm seed germination by the gibberellin/abscisic acid balance. Plant Biol. 2015, 17, 990-996.

8. Mauriat, M.; Moritz, T. Analyses of GA20ox- and GID1-over-expressing aspen suggest that gibberellins play two distinct roles in wood formation. Plant J. 2009, 58, 989-1003. 
9. Ragni, L.; Nieminen, K.; Pacheco-Villalobos, D.; Sibout, R.; Schwechheimer, C.; Hardtke, C.S. Mobile gibberellin directly stimulates Arabidopsis hypocotyl xylem expansion. Plant Cell 2011, 23, 1322-1336.

10. Biemelt, S.; Tschiersch, H.; Sonnewald, U. Impact of altered gibberellin metabolism on biomass accumulation, lignin biosynthesis, and photosynthesis in transgenic tobacco plants. Plant Physiol. 2004, 135, 254-265.

11. Zhong, R.; Lee, C.; Ye, Z.H. Evolutionary conservation of the transcriptional network regulating secondary cell wall biosynthesis. Trends Plant Sci. 2010, 15, 625-632.

12. Kim, S.G.; Lee, A.K.; Yoon, H.K.; Park, C.M. A membrane-bound NAC transcription factor NTL8 regulates gibberellic acid-mediated salt signaling in Arabidopsis seed germination. Plant J. Cell Mol. Biol. 2008, 55, 77-88.

13. Yuan, Y.; Wu, C.; Liu, Y.; Yang, J.; Huang, L. The Scutellaria baicalensis R2R3-MYB transcription factors modulates flavonoid biosynthesis by regulating GA metabolism in transgenic tobacco plants. PLOS ONE 2013, 8, e77275.

14. Ohashi-Ito, K.; Oda, Y.; Fukuda, H. Arabidopsis VASCULAR-RELATED NAC-DOMAIN6 directly regulates the genes that govern programmed cell death and secondary wall formation during xylem differentiation. Plant Cell 2010, 22, 3461-3473.

15. Zhong, R.; Lee, C.; Zhou, J.; McCarthy, R.L.; Ye, Z.H. A battery of transcription factors involved in the regulation of secondary cell wall biosynthesis in Arabidopsis. Plant Cell 2008, 20, 2763-2782.

16. Ko, J.H.; Kim, W.C.; Han, K.H. Ectopic expression of MYB46 identifies transcriptional regulatory genes involved in secondary wall biosynthesis in Arabidopsis. Plant J. 2009, 60, 649-665.

17. Pimenta Lange, M.J.; Lange, T. Gibberellin biosynthesis and the regulation of plant development. Plant Biol. 2006, 8, 281-290.

18. Arana, M.V.; Sanchez-Lamas, M.; Strasser, B.; Ibarra, S.E.; Cerdan, P.D.; Botto, J.F.; Sanchez, R.A. Functional diversity of phytochrome family in the control of light and gibberellin-mediated germination in Arabidopsis. Plant Cell Environ. 2014, 37, 2014-2023.

19. Rhie, Y.H.; Lee, S.Y.; Kim, K.S. Seed dormancy and germination in Jeffersonia dubia (Berberidaceae) as affected by temperature and gibberellic acid. Plant Biol. 2015, 17, 327-334.

20. Peng, J.; Harberd, N.P. The role of GA-mediated signalling in the control of seed germination. Curr. Opin. Plant Biol. 2002, 5, 376-381.

21. Sauret-Gueto, S.; Calder, G.; Harberd, N.P. Transient gibberellin application promotes Arabidopsis thaliana hypocotyl cell elongation without maintaining transverse orientation of microtubules on the outer tangential wall of epidermal cells. Plant J. Cell Mol. Biol. 2012, 69, 628-639.

22. Zhang, Y.; Liu, Z.; Wang, L.; Zheng, S.; Xie, J.; Bi, Y. Sucrose-induced hypocotyl elongation of Arabidopsis seedlings in darkness depends on the presence of gibberellins. J. Plant Physiol. 2010, 167, 1130-1136.

23. Jiang, S.; Xu, K.; Wang, Y.Z.; Ren, Y.P.; Gu, S. Role of GA3, GA4 and uniconazole- $P$ in controlling gravitropism and tension wood formation in Fraxinus mandshurica Rupr. var. japonica Maxim. seedlings. J. Int. Plant Biol. 2008, 50, 19-28.

24. Tokunaga, N.; Uchimura, N.; Sato, Y. Involvement of gibberellin in tracheary element differentiation and lignification in Zinnia elegans xylogenic culture. Protoplasma 2006, 228, 179-187. 
25. Wang, C.; Zhang, N.; Gao, C.; Cui, Z.; Sun, D.; Yang, C.; Wang, Y. Comprehensive transcriptome analysis of developing xylem responding to artificial bending and gravitational stimuli in Betula platyphylla. PLoS ONE 2014, 9, e87566.

26. Ye, Z.H.; Zhong, R. Molecular control of wood formation in trees. J. Exp. Bot. 2015, 66, 4119-4131.

27. Mitsuda, N.; Ohme-Takagi, M. NAC transcription factors NST1 and NST3 regulate pod shattering in a partially redundant manner by promoting secondary wall formation after the establishment of tissue identity. Plant J. 2008, 56, 768-778.

28. Ji, L.; Hu, R.; Jiang, J.; Qi, G.; Yang, X.; Zhu, M.; Fu, C.; Zhou, G.; Yi, Z. Molecular cloning and expression analysis of 13 NAC transcription factors in Miscanthus lutarioriparius. Plant Cell Rep. 2014, 33, 2077-2092.

29. Chen, X.; Lu, S.; Wang, Y.; Zhang, X.; Lv, B.; Luo, L.; Xi, D.; Shen, J.; Ma, H.; Ming, F. OsNAC2 encoding a NAC transcription factor affects plant height through mediating the gibberellic acid pathway in rice. Plant J. 2015, 82, 302-314.

30. Hussey, S.G.; Mizrachi, E.; Spokevicius, A.V.; Bossinger, G.; Berger, D.K.; Myburg, A.A. SND2, a NAC transcription factor gene, regulates genes involved in secondary cell wall development in Arabidopsis fibres and increases fibre cell area in Eucalyptus. BMC Plant Biol. 2011, 11, doi:10.1186/1471-2229-11-173.

31. McCarthy, R.L.; Zhong, R.; Ye, Z.H. MYB83 is a direct target of SND1 and acts redundantly with MYB46 in the regulation of secondary cell wall biosynthesis in Arabidopsis. Plant Cell Physiol. 2009, 50, 1950-1964.

32. Zhong, R.; Richardson, E.A.; Ye, Z.H. The MYB46 transcription factor is a direct target of SND1 and regulates secondary wall biosynthesis in Arabidopsis. Plant Cell 2007, 19, 2776-2792.

33. Zhao, K.; Bartley, L.E. Comparative genomic analysis of the R2R3 MYB secondary cell wall regulators of Arabidopsis, poplar, rice, maize, and switchgrass. BMC Plant Biol. 2014, 14, doi:10.1186/1471-2229-14-135.

34. Gubler, F.; Kalla, R.; Roberts, J.K.; Jacobsen, J.V. Gibberellin-regulated expression of a myb gene in barley aleurone cells: Evidence for Myb transactivation of a high-pI $\alpha$-amylase gene promoter. Plant Cell 1995, 7, 1879-1891.

35. Kim, W.C.; Kim, J.Y.; Ko, J.H.; Kang, H.; Han, K.H. Identification of direct targets of transcription factor MYB46 provides insights into the transcriptional regulation of secondary wall biosynthesis. Plant Mol. Biol. 2014, 85, 589-599.

36. Kim, W.C.; Ko, J.H.; Kim, J.Y.; Kim, J.M.; Bae, H.J.; Han, K.H. MYB46 directly regulates the gene expression of secondary wall-associated cellulose synthases in Arabidopsis. Plant J. 2013, 73, 26-36.

37. Liang, Y.K.; Xie, X.; Lindsay, S.E.; Wang, Y.B.; Masle, J.; Williamson, L.; Leyser, O.; Hetherington, A.M. Cell wall composition contributes to the control of transpiration efficiency in Arabidopsis thaliana. Plant J. Cell Mol. Biol. 2010, 64, 679-686.

38. Zhang, B.; Deng, L.; Qian, Q.; Xiong, G.; Zeng, D.; Li, R.; Guo, L.; Li, J.; Zhou, Y. A missense mutation in the transmembrane domain of CESA4 affects protein abundance in the plasma membrane and results in abnormal cell wall biosynthesis in rice. Plant Mol. Biol. 2009, 71, 509-524. 
39. Carroll, A.; Mansoori, N.; Li, S.; Lei, L.; Vernhettes, S.; Visser, R.G.; Somerville, C.; Gu, Y.; Trindade, L.M. Complexes with mixed primary and secondary cellulose synthases are functional in Arabidopsis plants. Plant Physiol. 2012, 160, 726-737.

40. Li, S.; Lei, L.; Gu, Y. Functional analysis of complexes with mixed primary and secondary cellulose synthases. Plant Signal. Behav. 2013, 8, e23179.

41. Gimeno-Gilles, C.; Lelievre, E.; Viau, L.; Malik-Ghulam, M.; Ricoult, C.; Niebel, A.; Leduc, N.; Limami, A.M. ABA-mediated inhibition of germination is related to the inhibition of genes encoding cell-wall biosynthetic and architecture: Modifying enzymes and structural proteins in Medicago truncatula embryo axis. Mol. Plant 2009, 2, 108-119.

42. Sundari, B.K.; Dasgupta, M.G. Isolation of developing secondary xylem specific cellulose synthase genes and their expression profiles during hormone signalling in Eucalyptus tereticornis. J. Genet. 2014, 93, 403-414.

43. Costa, M.A.; Collins, R.E.; Anterola, A.M.; Cochrane, F.C.; Davin, L.B.; Lewis, N.G. An in silico assessment of gene function and organization of the phenylpropanoid pathway metabolic networks in Arabidopsis thaliana and limitations thereof. Phytochemistry 2003, 64, 1097-1112.

44. Calvo, A.P.; Nicolas, C.; Nicolas, G.; Rodriguez, D. Evidence of a cross-talk regulation of a GA 20-oxidase (FsGA20oxl) by gibberellins and ethylene during the breaking of dormancy in Fagus sylvatica seeds. Physiol. Plant. 2004, 120, 623-630.

45. Marti, E.; Carrera, E.; Ruiz-Rivero, O.; Garcia-Martinez, J.L. Hormonal regulation of tomato gibberellin 20-oxidase1 expressed in Arabidopsis. J. Plant Physiol. 2010, 167, 1188-1196.

46. Rieu, I.; Ruiz-Rivero, O.; Fernandez-Garcia, N.; Griffiths, J.; Powers, S. J.; Gong, F.; Linhartova, T.; Eriksson, S.; Nilsson, O.; Thomas, S.G.; et al. The gibberellin biosynthetic genes AtGA20oxl and AtGA20ox2 act, partially redundantly, to promote growth and development throughout the Arabidopsis life cycle. Plant J. 2008, 53, 488-504.

47. Tian, J.; Du, Q.; Chang, M.; Zhang, D. Allelic variation in PtGA20Ox associates with growth and wood properties in Populus spp. PLoS ONE 2012, 7, e53116.

48. Zentella, R.; Zhang, Z.L.; Park, M.; Thomas, S.G.; Endo, A.; Murase, K.; Fleet, C.M.; Jikumaru, Y.; Nambara, E.; Kamiya, Y.; et al. Global analysis of della direct targets in early gibberellin signaling in Arabidopsis. Plant Cell 2007, 19, 3037-3057.

49. Ogawa, M.; Hanada, A.; Yamauchi, Y.; Kuwahara, A.; Kamiya, Y.; Yamaguchi, S. Gibberellin biosynthesis and response during Arabidopsis seed germination. Plant Cell 2003, 15, 1591-1604.

50. Mortazavi, A.; Williams, B.A.; McCue, K.; Schaeffer, L.; Wold, B. Mapping and quantifying mammalian transcriptomes by RNA-Seq. Nat. Methods 2008, 5, 621-628.

51. Pfaffl, M.W.; Horgan, G.W.; Dempfle, L. Relative expression software tool (RESTC) for group-wise comparison and statistical analysis of relative expression results in real-time PCR. Nucleic Acids Res. 2002, 30, doi:10.1093/nar/30.9.e36.

(C) 2015 by the authors; licensee MDPI, Basel, Switzerland. This article is an open access article distributed under the terms and conditions of the Creative Commons Attribution license (http://creativecommons.org/licenses/by/4.0/). 